\title{
Treatment options in nodular lymphocyte-predominant Hodgkin lymphoma
}

Dennis A Eichenauer ${ }^{* 1,2} \&$ Andreas Engert ${ }^{1,2}$
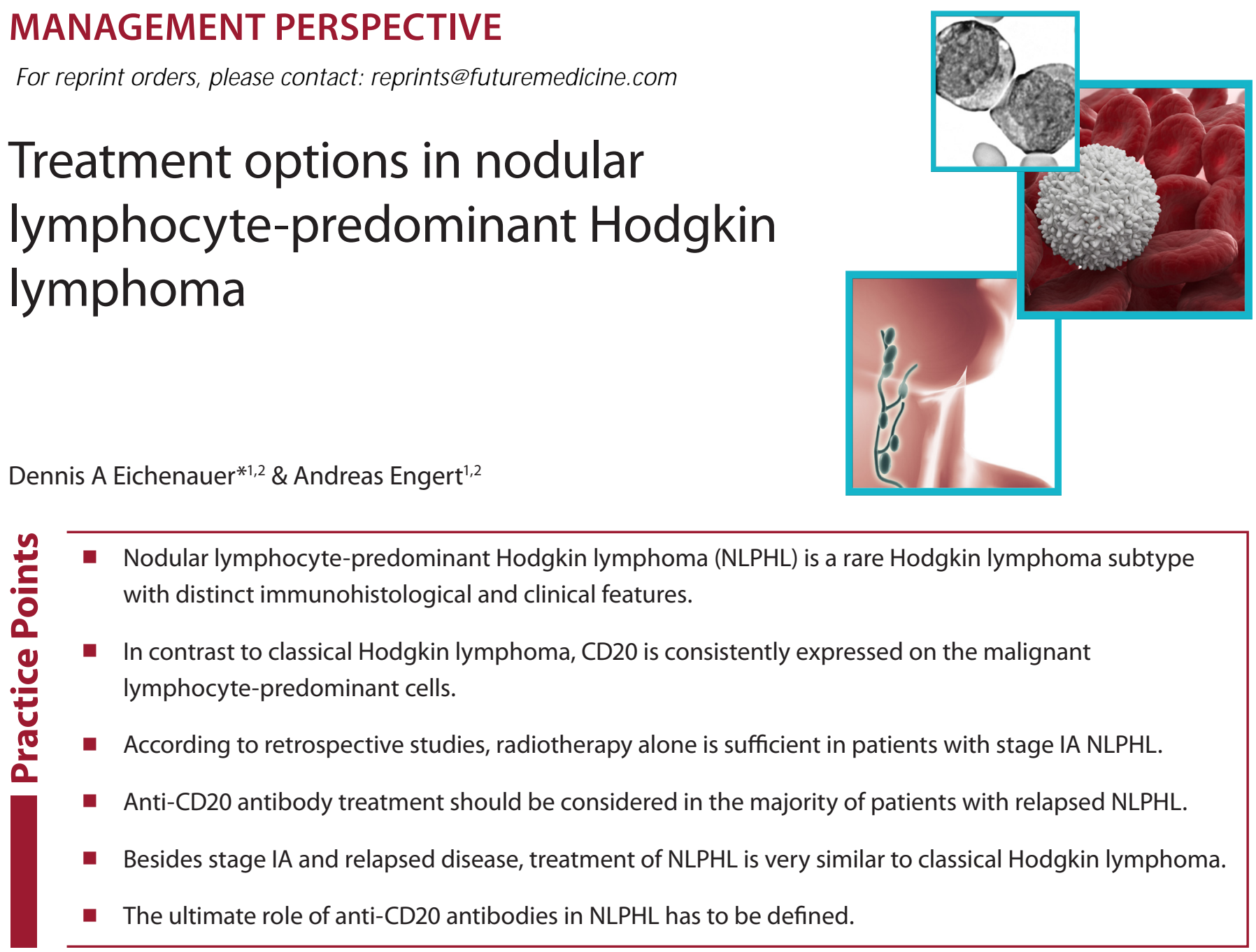

SUMMARY Nodular lymphocyte-predominant Hodgkin lymphoma accounts for approximately $5 \%$ of all Hodgkin lymphoma cases. Although this entity is characterized by immunohistological and clinical features such as a consistent expression of CD20 on the malignant lymphocyte-predominant cells and a more indolent clinical course resembling indolent non-Hodgkin lymphoma, treatment is often similar to classical Hodgkin lymphoma. Other approaches appear possible but data are scarce. Especially the ultimate role of anti-CD20 antibodies, the optimal first-line chemotherapy protocol and the value of high-dose chemotherapy followed by autologous stem cell transplantation in relapsed disease are undefined to date. The present article aims at summarizing and discussing the most recent publications on treatment options in nodular lymphocyte-predominant Hodgkin lymphoma.

Nodular lymphocyte-predominant Hodgkin lymphoma (NLPHL) represents approximately $5 \%$ of all Hodgkin lymphoma (HL) cases [1]. This entity substantially differs from the histological subtypes of classical HL (cHL) in terms of immunohistology, presentation and clinical course. As compared with Hodgkin and ReedSternberg cells in cHL, the disease-defining lymphocyte-predominant cells consistently express the B-cell marker CD20 while the antigens CD15 and CD30, which are both found on Hodgkin and Reed-Sternberg cells, can usually not be detected (Table 1 \& Figure 1) [2]. The majority of NLPHL patients are diagnosed with early favorable stages. After adequate treatment, these patients achieve long-term remission in 
Table 1. Staining characteristics of nodular lymphocyte-predominant Hodgkin

lymphoma and classical Hodgkin lymphoma.

\begin{tabular}{|lll|} 
& NLPHL & cHL \\
CD15 & - & + \\
CD20 & + & $+/-$ \\
CD30 & - & + \\
CD45 & + & - \\
EMA & + & - \\
\hline CHL: Classical Hodgkin lymphoma; NLPHL: Nodular \\
lymphocyte-predominant Hodgkin lymphoma.
\end{tabular}

approximately $90 \%$ of cases. In more advanced NLPHL, particularly late relapses are frequently observed. However, most relapses can be salvaged successfully so that overall survival (OS) is not impaired [3]. Limited data on specific treatment strategies for NLPHL are available mainly due to the low incidence. Thus, treatment is often very similar to cHL although different approaches appear possible in several clinical situations. This article critically reviews the most recent analyses on therapeutic options in newly diagnosed and relapsed NLPHL. In addition, possible future treatment strategies are discussed.

\section{Treatment of early favorable stages}

In contrast to $\mathrm{cHL}$, the majority of NLPHL patients are diagnosed with early favorable stages. Patients with stage IA disease are usually treated with involved-field radiotherapy (IF-RT) alone (Table 2) while patients with early favorable stages other than stage IA are treated with combined-modality approaches at most institutions. Since these treatment strategies result in excellent long-term OS rates, recently finished and ongoing studies aim at reducing the therapy-related acute and long-term toxicity without compromising the clinical outcome.

The German Hodgkin Study Group (GHSG) retrospectively analyzed 131 NLPHL patients diagnosed with stage IA disease without clinical risk factors. Extended-field RT (EF-RT; $\mathrm{n}=45)$, IF-RT $(\mathrm{n}=45)$ or combined-modality treatment (CMT; $\mathrm{n}=41$ ) were applied. Overall, 99\% of patients achieved a complete remission (CR); relapse rate was 5\% with no significant differences between the treatment approaches. At a median follow-up of 78 months for EF-RT, 17 months for IF-RT and 40 months for CMT, OS rates were 94, 100 and 96\%, respectively. Among patients treated with EF-RT or IF-RT, only 2.2\% experienced grade III/IV toxicity. By contrast, grade III/IV toxicity was observed in $48.8 \%$ of patients receiving CMT [4]. On the basis of this analysis, the GHSG adopted IF-RT alone as standard of care for patients with stage IA NLPHL without clinical risk factors.

Recent data from the USA are in line with the GHSG results. A total of 113 patients with stage I/II NLPHL treated with radiotherapy (RT) alone ( $\mathrm{n}=93)$, CMT $(\mathrm{n}=13)$ or chemotherapy alone $(n=7)$ at a single institution were retrospectively analyzed. After a median observation of 136 months among survivors, patients treated with RT alone had an excellent outcome. Additional chemotherapy did not lead to improved results. Chemotherapy alone was associated with an increased relapse rate. The extent of RT could be safely reduced from EF-RT to IF-RT without an impairment of treatment results. Secondary malignancies and cardiovascular disease were less frequent after IF-RT when compared with EF-RT. Patients with stage I disease had a superior progressionfree survival (PFS) when compared with stage II patients. However, this advantage in PFS did not translate into a better OS [5].

With the aim to prevent therapy-related late sequelae such as secondary malignancies and cardiovascular disease, the GHSG conducted a Phase II study evaluating the anti-CD20 antibody rituximab as single agent in 28 patients with newly diagnosed stage IA NLPHL presenting without clinical risk factors (Table 3). Response rate was $100 \%$. However, after a median follow-up of 43 months, seven patients had relapsed. Thus, single-agent rituximab appears to result in inferior tumor control as compared with RT alone and was not adopted as novel standard of care for the treatment of stage IA NLPHL within the GHSG [6].

Resection only, followed by watchful waiting was investigated by pediatric study groups. One larger retrospective analysis included 58 children aged 4-17 years who did not receive additional chemotherapy or RT after lymph node resection. The majority of patients (54 out of 58) had stage IA disease. After a median observation of 43 months, the PFS rate was $57 \%$ for all patients and $67 \%$ for patients in CR after surgery. All patients who were not in CR after lymph node resection eventually relapsed. The authors concluded that resection only may represent a treatment option in children with limited-stage NLPHL achieving a CR after surgical lymphadenectomy [7]. The question of whether this is also true for adult patients is unanswered since 
data addressing this issue are not available so far. However, given the increased relapse rate after resection only on the one hand and the excellent long-term results seen after limited-field RT on the other hand, this approach should not be common practice and should be restricted to carefully selected patients, such as those with risk factors for chemotherapy or RT, or those who refuse these treatment modalities.

Institutions such as the European Society for Medical Oncology (ESMO) and the National Comprehensive Cancer Network (NCCN) currently recommend IF-RT alone as standard of care for the treatment of adult patients with stage IA NLPHL without clinical risk factors $[1,8]$. The question of whether NLPHL patients diagnosed with early favorable stages other than stage IA are also sufficiently treated with RT alone is not yet answered. However, a recent analysis from Canada indicated that CMT approaches may represent the most suitable treatment in these patients. In this retrospective study, the outcome of 32 patients treated with RT alone between 1966 and 1993 was compared with the outcome of 56 patients treated with CMT approaches between 1993 and 2009. As a major finding, the study revealed a significantly better PFS for patients treated with CMT approaches [9]. Although this analysis has some limitations and results have to be interpreted with caution as patients were treated over a period of more than four decades, the improved tumor control with CMT approaches should be kept in mind when choosing treatment for patients with NLPHL in early favorable stages other than stage IA.

\section{Treatment of early unfavorable \& \\ advanced stages}

According to a large retrospective GHSG analysis, 16 and 21\% of NLPHL patients are diagnosed with early unfavorable and advanced stages, respectively. Usually, treatment is very similar to cHL consisting of CMT approaches for early unfavorable stages and six to eight cycles of chemotherapy followed by localized RT to larger residual lymphoma for advanced stages. The chemotherapy protocols most often used are ABVD (adriamycin, bleomycin, vinblastine and dacarbazine) or ABVD-like protocols and escalated BEACOPP (bleomycin, etoposide, adriamycin, cyclophosphamide, vincristine, procarbazine and prednisone). As revealed by the GHSG analysis, the clinical outcome of NLPHL patients after treatment with these protocols is comparable with the outcome of cHL patients [3]. Nonetheless, it is a matter of debate whether alkylator-based chemotherapy protocols may represent the more suitable treatment for more advanced NLPHL when compared with ABVD or ABVD-like protocols. The Cancer and Leukemia Group B retrospectively evaluated 37 patients with advanced NLPHL treated with either MOPP (mechlorethamine, vincristine, procarbazine and prednisone) and MOPP/ABVD or ABVD/EVA (etoposide, vinblastine and adriamycin). As a result, the relapse rate after ABVD/EVA was 75\% compared with only 32\% after MOPP or MOPP/ABVD indicating a superior tumor control after alkylatorbased chemotherapy [10]. First data on the use of R-CHOP (rituximab, cyclophosphamide, adriamycin, vincristine and prednisone) are also promising. A total of 15 patients (four stage I/II patients and 11 stage III/IV patients) were treated with this protocol at a single institution and outcome was retrospectively analyzed; response rate was $100 \%$. After a median follow-up of 42 months, no patient had relapsed [11]. However, results from prospective studies confirming these results are necessary to draw final conclusions.

In summary, treatment with standard HL protocols such as ABVD and BEACOPP optionally followed by RT is safe and effective in early unfavorable and advanced NLPHL [3,12]. Thus, they represent the standard of care at most

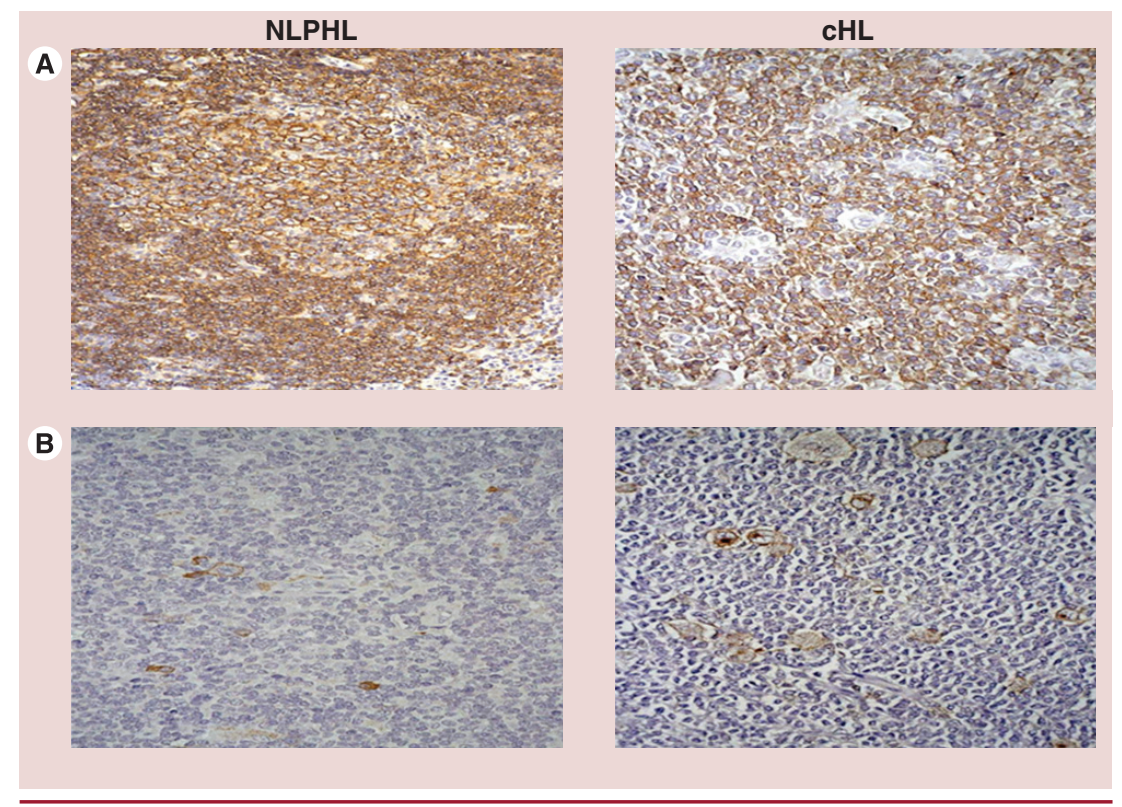

Figure 1. CD20 (A) and CD30 (B) staining in nodular lymphocyte-predominant Hodgkin lymphoma and classical Hodgkin lymphoma.

cHL: Classical Hodgkin lymphoma; NLPHL: Nodular lymphocyte-predominant Hodgkin lymphoma. 
Table 2. Radiotherapy alone for the treatment of newly diagnosed nodular lymphocyte-predominant Hodgkin lymphoma.

\begin{tabular}{|c|c|c|c|c|}
\hline Stages included & RT fields included & $\mathbf{n}$ & PFS & Ref. \\
\hline $\begin{array}{l}\text { Stage IA without } \\
\text { RF }\end{array}$ & IF-RT, EF-RT & 90 & $\begin{array}{l}\text { 2-year PFS: } \\
\text { 100\% for EF-RT } \\
92 \% \text { for IF-RT }\end{array}$ & [4] \\
\hline Stage I/II & $\begin{array}{l}\text { Limited, regional, } \\
\text { extended }\end{array}$ & 93 & $\begin{array}{l}\text { 10-year PFS: } \\
89 \% \text { for stage I } \\
72 \% \text { for stage II }\end{array}$ & [5] \\
\hline Stage I/II & $\begin{array}{l}\text { Mantle field and } \\
\text { variants, inverted } \\
\text { Y-field and variants, } \\
\text { TLNI }\end{array}$ & 202 & $\begin{array}{l}\text { 15-year PFS: } \\
84 \% \text { for stage I } \\
73 \% \text { for stage II }\end{array}$ & [19] \\
\hline
\end{tabular}

institutions. Other approaches such as R-CHOP are also feasible and active but mature prospective data defining their ultimate role are pending.

The risk of transformation into aggressive B-cell non-HL

In NLPHL patients presenting with clinical signs of relapse, a lymph node biopsy should be obtained whenever possible. This is due to an increased risk of transfomation from NLPHL into aggressive B-cell non-HL (B-NHL), T-cell-rich B-NHL in particular. Some analyses addressing this issue were recently performed. A registry-based analysis comprising 164 patients diagnosed with NLPHL between 1973 and 2003 came from France. At a median follow-up of 9.5 years for survivors, 66 patients had lymphoma recurrence; and 19 of them presented with histological transformation into aggressive B-NHL. The median time between the initial NLPHL diagnosis and the occurrence of transformation was 4.7 years; the cumulative 10-year transformation rate was $12 \%$. Patients with transformation were either treated with conventional chemotherapy (10 out of 19) or high-dose chemotherapy followed by autologous stem cell transplantation (ASCT; 9 out of 19) and thus more aggressive than patients who relapsed with NLPHL histology. However, patients with transformed lymphoma had a poorer prognosis in comparison with patients who had NLPHL histology at relapse [13].

A second report from Canada using the British Columbia Cancer Agency (BCCA) database included a total of 95 patients initially diagnosed with NLPHL. Transformation into aggressive B-NHL occurred in 13 of them; the median time to transformation was 8.1 years. The actuarial risk for the development of transformed lymphoma after initial diagnosis of NLPHL was 5, 7, 15, 31 and 36\% after 5, 10, 15, 20 and 25 years, respectively. Similar to the French report, prognosis after diagnosis of aggressive B-NHL was worse than expected after NLPHL relapse. Despite being treated with multiagent chemotherapy mostly followed by high-dose chemotherapy and ASCT, 10-year estimates for PFS and OS were only 52 and 62\%, respectively, for patients with transformed lymphoma [14].

Given these data, a rebiopsy to exclude transformation into aggressive B-NHL should be mandatory in patients initially treated for NLPHL and presenting with clinical signs of relapse.

\section{Treatment of relapsed NLPHL}

At present, standard treatment for histologically confirmed relapsed NLPHL is undefined. However, high-dose chemotherapy followed by ASCT - the standard of care for relapsed cHL - may not represent the optimal treatment for the majority of NLPHL patients with disease recurrence. Particularly in patients with low tumor burden,

Table 3. Single-agent rituximab for the treatment of nodular lymphocyte-predominant Hodgkin lymphoma.

\begin{tabular}{|c|c|c|c|c|c|c|}
\hline Disease status & $\begin{array}{l}\text { Stages } \\
\text { included }\end{array}$ & $\begin{array}{l}\text { Rituximab } \\
\text { schedule }\end{array}$ & $\mathbf{n}$ & $\begin{array}{l}\text { Response } \\
\text { rate (\%) }\end{array}$ & PFS & Ref. \\
\hline Untreated & IA without RF & Standard & 28 & 100 & $81.4 \%$ at $36 \mathrm{~m}$ & [6] \\
\hline Untreated & All stages & $\begin{array}{l}\text { Standard/ } \\
\text { extended }\end{array}$ & $\begin{array}{l}S=10 \\
E=9\end{array}$ & 100 & $\begin{array}{l}\text { Median PFS: } \\
\mathrm{S}=50 \mathrm{~m} \\
\mathrm{E}=67 \mathrm{~m}\end{array}$ & [20] \\
\hline $\begin{array}{l}\text { Untreated/ } \\
\text { relapsed }\end{array}$ & All stages & Standard & $\begin{array}{l}U=12 \\
R=10\end{array}$ & 100 & $\begin{array}{l}\text { Median PFS: } \\
10.2 \mathrm{~m}\end{array}$ & [15] \\
\hline Relapsed & All stages & Standard & 15 & 94 & Median PFS: 33 m & [16] \\
\hline
\end{tabular}


single-agent anti-CD20 antibody treatment appears to represent the appropriate approach.

This suggestion is supported by prospective data on the use of rituximab in relapsed NLPHL (Table 3). The Stanford group conducted a Phase II study including 12 patients with newly diagnosed NLPHL and ten patients with relapsed disease. Treatment consisted of four weekly rituximab infusions at a dose of $375 \mathrm{mg} / \mathrm{m}^{2}$. Response rate was $100 \%$. At a median follow-up of 13 months for the whole patient group, disease progression was seen in three out of the ten patients who had already received treatment prior to study entry [15].

Results from a GHSG Phase II study comprising 15 patients with relapsed NLPHL were similar. Within this study, patients also received four weekly standard doses of rituximab. Response rate was $94 \%$. After a median observation of 63 months, the median time to progression was 33 months, the median OS was not reached, and only one patient had died [16]. The tolerability of the antibody was excellent in both studies. Thus, single-agent anti-CD20 antibody treatment should at least be considered in patients with more localized NLPHL recurrence. This is true for both patients who are anti-CD20 antibody naive and patients who had previously received and achieved remission upon anti-CD20 antibody treatment as repeated responses have been observed in the study from the Stanford group.

In patients relapsing with more disseminated disease, aggressive approaches including conventional multiagent chemotherapy or high-dose chemotherapy followed by ASCT may be more appropriate. However, according to the limited available data on high-dose chemotherapy followed by ASCT in relapsed NLPHL, tumor control with this modality does not appear to be better than with rituximab. A retrospective analysis from the USA including 19 patients with relapsed NLPHL who were treated with high-dose chemotherapy followed by ASCT reported 5-year OS and PFS rates of only 56 and $40 \%$ [17]. By contrast, a recent study from the MD Anderson Cancer Center including 18 patients with confirmed NLPHL at relapse reported 5-year OS and event-free survival rates of 73 and $61 \%$, respectively, with this modality [18]. The improved clinical outcome in the latter report may in part be attributed to the relevant proportion of patients treated with rituximab as part of the conditioning regimen prior to ASCT.

In conclusion, given the relevant toxicity and the potentially reduced efficacy of high-dose chemotherapy followed by ASCT in relapsed NLPHL on the one hand and the good tolerability and the excellent response rates observed with singe-agent rituximab on the other hand, antiCD20 antibody treatment represents a reasonable choice for the majority of patients with relapsed NLPHL. However, patients with more advanced disease at relapse may be candidates for high-dose chemotherapy followed by ASCT. The efficacy of this treatment approach may be improved by implementing rituximab or follow-up products into salvage and conditioning regimens.

\section{Conclusion \& future perspective}

NLPHL represents a subtype of HL with distinct pathological and clinical characteristics. In patients diagnosed with stage IA disease without clinical risk factors and in patients with relapsed disease, treatment often differs from the standard approaches in cHL. Stage IA patients are sufficiently treated with RT alone and single-agent anti-CD20 antibody treatment results in longterm remission in a relevant proportion of patients with relapsed NLPHL $[4,5,15,16]$. In early unfavorable and advanced stages, treatment is very similar to $\mathrm{cHL}$. Additional prospective and retrospective studies are necessary to optimize the treatment of NLPHL. For instance, the clinical outcome may be improved by implementing rituximab or follow-up products into current standard protocols. In return, it might become possible to reduce the doses of conventional chemotherapeutics without compromising efficacy. Thereby, the risk of developing severe late effects such as heart and lung failure or infertility may decrease substantially. The possibility of a further reduction of RT doses and fields should also be evaluated as RT-associated late sequelae such as secondary solid tumors or hypothyroidism are frequently observed in longterm survivors. In relapsed NLPHL, more data on the use of anti-CD20 antibodies as single agents, as well as high-dose chemotherapy followed by ASCT, are required.

Financial \& competing interests disclosure

The authors have no relevant affiliations or financial involvement with any organization or entity with a financial interest in or financial conflict with the subject matter or materials discussed in the manuscript. This includes employment, consultancies, honoraria, stock ownership or options, expert testimony, grants or patents received or pending, or royalties.

No writing assistance was utilized in the production of this manuscript. 


\section{References}

Papers of special note have been highlighted as:

- of interest

-1. of considerable interest

1 Eichenauer DA, Engert A, Dreyling M. Hodgkin's lymphoma: ESMO clinical practice guidelines for diagnosis, treatment and follow-up. Ann. Oncol. 22(Suppl. 6), vi55-vi58 (2011).

2 Nogova L, Rudiger T, Engert A. Biology, clinical course and management of nodular lymphocyte-predominant Hodgkin lymphoma. Hematol. Am. Soc. Hematol. Educ. Program 266-272 (2006).

3 Nogova L, Reineke T, Brillant C et al. Lymphocyte-predominant and classical Hodgkin's lymphoma: a comprehensive analysis from the German Hodgkin Study Group. J. Clin. Oncol. 26(3), 434-439 (2008).

-1 Largest analysis on characteristics and outcome of patients with nodular lymphocyte-predominant Hodgkin lymphoma (NLPHL) published to date. Important information on clinical differences between NLPHL and classical Hodgkin lymphoma are provided.

4 Nogova L, Reineke T, Eich HT et al. Extended field radiotherapy, combined modality treatment or involved field radiotherapy for patients with stage IA lymphocyte-predominant Hodgkin's lymphoma: a retrospective analysis from the German Hodgkin Study Group (GHSG) Ann. Oncol. 16(10), 1683-1687 (2005).

5 Chen RC, Chin MS, Ng AK et al. Early-stage, lymphocyte-predominant Hodgkin's lymphoma: patient outcomes from a large, single-institution series with long follow-up. J. Clin. Oncol. 28(1), 136-141 (2010).

- Large retrospective single-center analysis on radiotherapy alone in patients with newly diagnosed early-stage NLPHL. Importantly, long-term follow-up data are provided.

6 Eichenauer DA, Fuchs M, Pluetschow A et al. Phase 2 study of rituximab in newly diagnosed stage IA nodular lymphocyte- predominant Hodgkin lymphoma: a report from the German Hodgkin Study Group. Blood 118(16), 4363-4365 (2011).

7 Mauz-Korholz C, Gorde-Grosjean S, Hasenclever $\mathrm{D}$ et al. Resection alone in 58 children with limited stage, lymphocytepredominant Hodgkin lymphoma-experience from the European network group on pediatric Hodgkin lymphoma. Cancer 110(1), 179-185 (2007).

8 Hoppe RT, Advani RH, Ai WZ et al. Hodgkin lymphoma, version 2.2012 featured updates to the NCCN guidelines. J. Natl Compr. Canc. Netw. 10(5), 589-597 (2012).

9 Savage KJ, Skinnider B, Al-Mansour M, Sehn LH, Gascoyne RD, Connors JM. Treating limited-stage nodular lymphocyte predominant Hodgkin lymphoma similarly to classical Hodgkin lymphoma with ABVD may improve outcome. Blood 118(17), 4585-4590 (2011).

10 Canellos GP, Mauch P. What is the appropriate systemic chemotherapy for lymphocyte-predominant Hodgkin's lymphoma? J. Clin. Oncol. 28(1), e8 (2010).

11 Fanale MA, Lai CM, McLaughlin P et al. Outcomes of nodular lymphocyte predominant Hodgkin's lymphoma (NLPHL) patients treated with R-CHOP. ASH Annu. Meeting Abstracts 116(21), 2812 (2010).

12 Xing KH, Connors JM, Al-Mansour M, Gascoyne RD, Skinnider B, Savage KJ. The outcome of advanced stage nodular lymphocyte predominant Hodgkin's lymphoma (NLPHL) compared with classical Hodgkin's lymphoma (CHL): a matched pair analysis. ASH Annu. Meeting Abstracts 120(21), 1531 (2012).

13 Biasoli I, Stamatoullas A, Meignin V et al. Nodular, lymphocyte-predominant Hodgkin lymphoma: a long-term study and analysis of transformation to diffuse large B-cell lymphoma in a cohort of 164 patients from the Adult Lymphoma Study Group. Cancer 116(3), 631-639 (2010).

14 Al-Mansour M, Connors JM, Gascoyne RD, Skinnider B, Savage KJ. Transformation to aggressive lymphoma in nodular lymphocyte- predominant Hodgkin's lymphoma. J. Clin. Oncol. 28(5), 793-799 (2010).

- Analysis on the risk of transformation from NLPHL into aggressive B-cell non-Hodgkin lymphoma. The results of this study underscore the importance of rebiopsies in patients presenting with clinical signs of relapse.

15 Ekstrand BC, Lucas JB, Horwitz SM et al. Rituximab in lymphocyte-predominant Hodgkin disease: results of a Phase 2 trial. Blood 101(11), 4285-4289 (2003).

16 Schulz H, Rehwald U, Morschhauser F et al. Rituximab in relapsed lymphocytepredominant Hodgkin lymphoma: long-term results of a Phase 2 trial by the German Hodgkin Lymphoma Study Group (GHSG). Blood 111(1), 109-111 (2008).

- Analysis providing data on the activity of single-agent rituximab in patients with relapsed NLPHL.

17 Bierman P, Naushad H, Loberiza F et al. High-dose chemotherapy followed by autologous hematopoietic stem cell transplantation (AHSCT) for lymphocyte predominant Hodgkin's disease. ASH Annu. Meeting Abstracts 108(11), 3061 (2006).

18 Karuturi M, Hosing C, Fanale M et al. Highdose chemotherapy and autologous stem cell transplantation for nodular lymphocytepredominant Hodgkin lymphoma. Biol. Blood Marrow Transplant. 19(6), 991-994 (2013).

19 Wirth A, Yuen K, Barton M et al. Long-term outcome after radiotherapy alone for lymphocyte-predominant Hodgkin lymphoma: a retrospective multicenter study of the Australasian Radiation Oncology Lymphoma Group. Cancer 104(6), 1221-1229 (2005).

20 Advani RH, Horning SJ, Hoppe RT et al. Frontline therapy of nodular lymphocyte predominant Hodgkin lymphoma with rituximab: the Stanford University experience. ASH Annu. Meeting Abstracts 118(21), 2686 (2011). 